\title{
Algorithms and Insights for RaceTrack
}

\author{
Michael A. Bekos ${ }^{1}$, Till Bruckdorfer ${ }^{2}$, Henry Förster ${ }^{3}$, \\ Michael Kaufmann ${ }^{4}$, Simon Poschenrieder ${ }^{5}$, and Thomas Stüber ${ }^{6}$
}

$\begin{array}{ll}1 & \text { Wilhelm-Schickard-Institut für Informatik, Universität Tübingen, Germany } \\ 2 & \text { Wilhelm-Schickard-Institut für Informatik, Universität Tübingen, Germany } \\ 3 & \text { Wilhelm-Schickard-Institut für Informatik, Universität Tübingen, Germany } \\ 4 & \text { Wilhelm-Schickard-Institut für Informatik, Universität Tübingen, Germany } \\ 5 & \text { Wilhelm-Schickard-Institut für Informatik, Universität Tübingen, Germany } \\ 6 & \text { Wilhelm-Schickard-Institut für Informatik, Universität Tübingen, Germany }\end{array}$

\begin{abstract}
We discuss algorithmic issues on the well-known paper-and-pencil game RACETRACK. On a very simple track called Indianapolis, we introduce the problem and simple approaches, that will be gradually refined. We present and experimentally evaluate efficient algorithms for single player scenarios. We also consider a variant where the parts of the track are known as soon as they become visible during the race.
\end{abstract}

1998 ACM Subject Classification G.2.2 Graph Theory

Keywords and phrases Racetrack, State-graph, complexity

Digital Object Identifier 10.4230/LIPIcs.FUN.2016.6

\section{Introduction}

RACETRACK is a paper-and-pencil game that simulates a car race. The game is played by two or more players on a squared sheet of paper, where pencil-lines keep track of cars' moves [8]. Cars move from one point to a new point of the underlying grid along a track, whose boundary is freehand drawn on the sheet of paper. Each move is subject to some rules that aim in simulating a car with a certain inertia and physical limits on traction. The rules are relatively simple and so the game is very popular even for pupils:

R.1: No two cars can simultaneously occupy the same grid point.

R.2: A grid point occupied by a car must lie within the track. In addition, the trajectory of a car must not intersect the boundaries of the track.

R.3: At each move, a car can change its speed by at most one unit of distance at the horizontal and/or at the vertical direction.

Initially, the cars are aligned along the so-called start line. At each turn, a player moves her car along the track according to rules R.1-3. The first car crossing the so-called finish line in a specific direction wins. Note that rule R.3 is also referred to as eight-neighbours rule. The reason is the following. Each move of a car can be represented by a 2-dimensional vector, e.g., a move two units to the right and four units downwards corresponds to vector $(2,-4)$. Hence, at each move each coordinate of this vector is allowed to change by \pm 1 (simulating acceleration and deceleration, respectively), which gives rise to nine possible new grid points for the next move (eight of which are neighboring the previous one). Note that the game also serves as an educational tool for teaching vectors.

From an algorithmic point of view, the state of a car can be nicely encoded by a quadruple $\left(x, y, s_{x}, s_{y}\right)$, where $(x, y)$ denotes the position of the car on the track and $\left(s_{x}, s_{y}\right)$ correspond

(i) (i) Michael A. Bekos, Till Bruckdorfer, Henry Förster, Michael Kaufmann, Simon Poschenrieder and Thomas Stüber; licensed under Creative Commons License CC-BY 8th International Conference on Fun with Algorithms (FUN 2016). Editors: Erik D. Demaine and Fabrizio Grandoni; Article No. 6; pp. 6:1-6:14 Leibniz International Proceedings in Informatics LIPICS Schloss Dagstuhl - Leibniz-Zentrum für Informatik, Dagstuhl Publishing, Germany 
to its speed. By the eight-neighbours rule, it follows that subsequent states $\left(x, y, s_{x}, s_{y}\right)$ and $\left(x^{\prime}, y^{\prime}, s_{x}^{\prime}, s_{y}^{\prime}\right)$ must comply with the following two conditions: (i) $x^{\prime}=x+s_{x}^{\prime}$ and $y^{\prime}=y+s_{y}^{\prime}$, (ii) $\left|s_{x}-s_{x}^{\prime}\right| \leq 1$ and $\left|s_{y}-s_{y}^{\prime}\right| \leq 1$. The goal is to compute the minimum number of subsequent states from a certain starting position to the finish line.

The remainder of this paper is structured as follows: Section 2 overviews related work. In Section 3, we present a simple formulation of the problem as a shortest-path problem. We employ this simple formulation in order to develop a more efficient algorithm for a discretized version of the problem, where the boundaries of the track are rectilinear line segments (see Section 4). In Section 5, we consider a variant where the parts of the track are known as soon as they become visible during the race and we propose different strategies to drive safely but as fast as possible along the track. We experimentally evaluate our algorithms in Section 6 . We conclude in Section 7 with open problems.

\section{Previous work}

To the best of our knowledge, the first reference to RACETrACK is due to Gardner [3] back in 1973, who seemed to learn about this game from a Swiss colleague. Note that the game is known under several names such as Vector Formula, Vector Rally, Vector Race, Graph Racers, PolyRace, Paper and pencil racing, or the Graph paper race game [8].

Next to Gardner, Erickson [2] discussed RaceTrack in his blog, where he considered the classic problem on the grid graph to be polynomial time solvable and conjectured the computational problem to be PSPACE-complete when the boundary of the tracks are given by sequences of line segments, which might make the size of the output exponential.

Although the running time for an algorithm is polynomial in the number of allowed grid points $\left(O\left(n^{3}\right)\right.$ as stated by Erickson), Holzer and McKenzie [5] considered the decision problem for a winning strategy, distinguished variants where the boundary might be touched or not, and showed an NL-completeness result for the single-player variant. For the original 2-player variant, they show P-completeness, and summarize that "RACETRACK is an example of a game that is interesting to play despite the fact that deciding the existence of a winning strategy is most likely not NP-hard".

Schmid [7] presents a BFS-based algorithm to find the fastest path of a car through a twodimensional track. Olsson and Tarandi [6] describe an implementation of a genetic algorithm for RaCETrack. Ahlmann-Ohlsen [1] apply binary decisions diagrams to RACETraCK.

\section{A first approach}

The simplest scenario is to determine the minimum number of moves from a starting position along the track to the finish line, in the case where there exists only one car. Even though this problem sounds like a simple shortest-path problem, a closer look will reveal several problems that we will shortly analyze in more detail.

Following Erickson's approach, we assume that the underlying grid is of size $n \times n$. This implies that the maximum speed $s_{\max }$ of a car (either at the horizontal or at the vertical direction), which moves from one side of the track towards its opposite side cannot be more than $O(\sqrt{n})$, as the distance in one direction that is covered by the car with maximum speed $s_{\max }$ is at least $2 \sum_{i=0}^{s_{\max }-1} i+s_{\max }$, which is at most $n$.

Let $G=(V, E)$ be the so-called state graph which has a vertex for each possible state of a car and an edge between two states if and only if they are subsequent. By the eight-neighbors rule, $G$ has out-degree at most 9 . Hence, $|E|=O(|V|)$. Since the underlying grid is of size 
$O(n) \times O(n)$ and each speed component ranges from 0 to $O(\sqrt{n})$ at most, the size of $G$ is $O\left(n^{3}\right)$. To determine the minimum number of moves from a certain starting position to the finish line, we can simply perform a BFS algorithm on $G$. So, the complexity of this algorithm is $O\left(n^{3}\right)$, as independently observed by Erickson [2] and Schmid [7].

\section{$4 \quad$ A more efficient approach}

The algorithm described in the previous section shows that the problem of determining the minimum number of moves from a certain starting position to the finish line is polynomial time tractable, in the case of a single car (that is, rule R.1 is redundant). However, it is not difficult to observe that the size of the state graph can be too large even for relatively small tracks, which suggests that this first simple algorithm might not be really useful in practice. Motivated by this observation, in the following we will suggest an alternative approach that seeks to drastically reduce the size of the state graph (and therefore improve the usefulness of the corresponding shortest-path-based algorithm), assuming that the boundaries of the track are rectilinear line segments of particular lengths. Note that this is a quite reasonable assumption, as such tracks are quite common in RACETRACK.

In order to keep our presentation simple, we initially consider a simple rectangular-shaped track, that we call InDIANAPOLIS (see Figure 1). Later in this section, we show to which extend we can generalize our approach. INDIANAPOLIS track consists of four track segments of uniform width $W$ that overlap exactly at the corner regions (dashed drawn in Figure 1). The horizontal track segments are of length $L_{1}$, while the vertical track segments are of height $L_{2}$, as illustrated in Figure 1. For simplicity, we assume that the parts of the track segments between the corner regions are at least of length $W$. Note that the time complexity of the algorithm of the previous section adjusted to INDIANAPOLIS is $O\left(W^{3 / 2}\left(L_{1}+L_{2}\right)^{3 / 2}\right)$, because the underlying grid is of size $O\left(W\left(L_{1}+L_{2}\right)\right)$ and speed $s_{x}$ ranges between 0 and $O\left(\sqrt{L_{1}}\right)$ when $s_{y}$ ranges between 0 and $O(\sqrt{W})$ (horizontal track segment) and when $s_{y}$ ranges between 0 and $O\left(\sqrt{L_{2}}\right)$ then $s_{x}$ ranges between 0 and $O(\sqrt{W})$ (vertical track segment).

\subsection{Shrinking the size of the state graph for Indianapolis track}

In order to shrink the size of the state graph, we will determine relatively small sets of points (and appropriate speed components for these points), so that a car must necessarily "land" on at least one of the points of each set in order to reach the finish line. We refer to such points as landing points. We also say that neighboring landing points form a so-called landing region. Intuitively, a landing point should be in or close to a corner region of the track. The idea underneath is that a car cannot reach the finish line without first passing through every corner of the track, in which its direction has to be changed (from horizontal to vertical or vice versa). Hence, our landing regions are nearly subsets of corner regions (which are of size $W \times W$; refer to the dashed-drawn squares of Figure 1).

Our approach is outlined as follows: First, we determine the set of potential landing points at each corner region of the track. Then, we determine the minimum number of moves between points of consecutive landing regions (which is a kind of all-pairs shortest-paths between consecutive landing regions). In a third step, we perform a weighted shortest-path search to compute the actual minimum number of moves from a starting position (through all computed landing regions) to the finish line. 


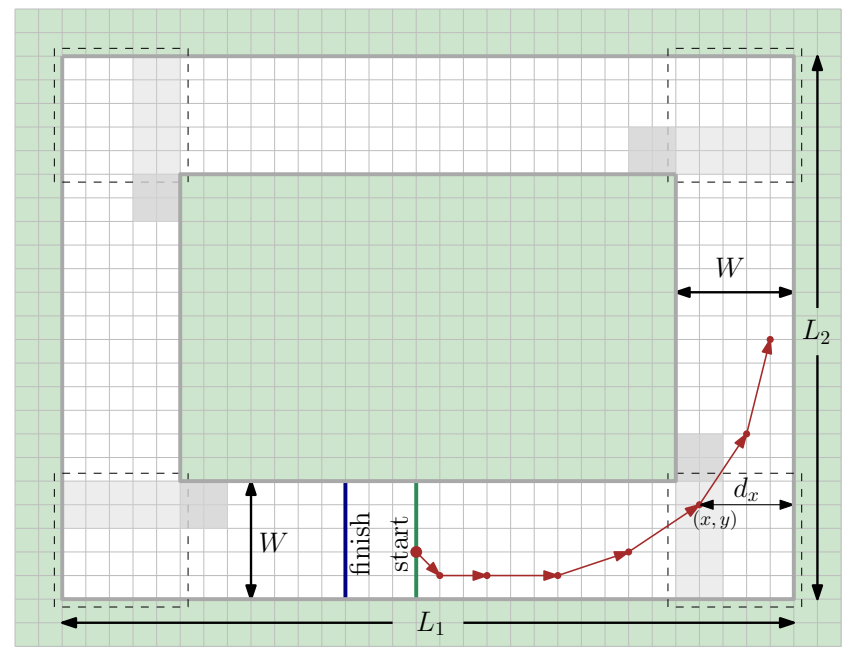

Figure 1 Illustration of the INDIANAPOLIS track: The corner regions are within the dashed-drawn squares. The landing regions are highlighted in gray.

\section{Determining the landing points}

Let $p=(x, y)$ be a potential landing point close to a corner of the track. If a car is at this point, then it must be able to change its direction, say w.l.o.g. from horizontal-right to vertical-up. Let $q=\left(x, y, s_{x}, s_{y}\right)$ be the state of the car at landing point $p$ and let $d_{x}$ be the distance of point $p$ to the next (vertical) side of the track. Observe that if $d_{x}$ is too small, then the car will crash. To avoid this scenario, the following relation must hold: $\sum_{i=1}^{s_{x}-1} i \leq d_{x}$. In the case where $d_{x}=W$, it follows that $s_{x} \leq \sqrt{2 W}$. Consider now the set of points within the vertical strip of width $\sqrt{2 W}$, whose left side coincides with the interior corner of the corresponding corner region (a part of it is highlighted in gray in Figure 1). From the above calculations, it follows that if a car passes through this area in the horizontal direction without landing on it, then it will crash on the next (vertical) side of the track. On the other hand, if a car has a speed $\leq \sqrt{2 W}$ exactly before this vertical strip, then it will land on this strip and it will be able to avoid crashing.

So, this vertical strip is a good candidate for serving as a potential landing region. Next, we will further constrain its height. By the same arguments as before, $s_{y}$ cannot be larger than $\sqrt{2 W}$, if the car lands somewhere at the intersection of the vertical strip and the corner region and moves upwards. Alternatively, the car might avoid landing at the corner region, if it moves in one step from the left side of the corner region to some point above it. In this case, $s_{y} \leq \sqrt{2 W}+1$ and $s_{x} \leq \sqrt{2 W}$ hold. It follows that the possible landing points for this case are in a rectangle of size $\sqrt{2 W} \times(\sqrt{2 W}+1)$ whose bottom left corner coincides with the interior corner of the corresponding corner region. Hence, the landing region of this corner is formed by the union of this rectangle and the rectangle of size $\sqrt{2 W} \times W$ below it. Note that some points associated with certain "low" speeds in the landing regions cannot be the first ones to land on. So, they can be neglected.

\section{Moving from a landing point to a new landing point}

We describe how to determine the minimum number of moves from one landing point $p$ to another landing point $p^{\prime}$ of the next landing region. Let $q=\left(x, y, s_{x}, s_{y}\right)$ and $q^{\prime}=$ $\left(x^{\prime}, y^{\prime}, s_{x}^{\prime}, s_{y}^{\prime}\right)$ be the corresponding states of the car at points $p$ and $p^{\prime}$, respectively. Since 
$p$ and $p^{\prime}$ belong to consecutive landing regions along the track, the movements in $x$ - and $y$-directions can be handled independently. The only restriction is that the number of moves in one direction must be the same as the number of moves in the other direction. First, we determine the minimum numbers $\tau_{x}$ and $\tau_{y}$ of moves in $x$ - and $y$-direction, respectively. Then, we "synchronize" them (by enlarging the smaller of the two), so to become same but still minimal.

In the following, we describe the computation for $\tau_{x}$. The computation of $\tau_{y}$ is symmetric. Let $\left|x-x^{\prime}\right|=\delta_{x}$ be the horizontal distance between $p$ and $p^{\prime}$. We assume w.l.o.g. that $s_{x} \leq s_{x}^{\prime}$. The case where $s_{x}>s_{x}^{\prime}$ is symmetric. Observe that $\tau_{x}$ is at least $s_{x}^{\prime}-s_{x}$, because we have to perform at least $s_{x}^{\prime}-s_{x}$ moves with speed increasing from $s_{x}$ to $s_{x}^{\prime}$. The distance $\alpha_{x}$ covered during theses moves is $\left(s_{x}+1\right)+\ldots+\left(s_{x}+\left(s_{x}^{\prime}-s_{x}\right)\right)=\left(s_{x}^{\prime}-s_{x}\right)\left(s_{x}^{\prime}+s_{x}+1\right) / 2$. If $\alpha_{x}=\delta_{x}$, then $\tau_{x}$ must be equal to $s_{x}^{\prime}-s_{x}$. Otherwise, we consider the cases $\alpha_{x}<\delta_{x}$ and $\alpha_{x}>\delta_{x}$ separately. Before we proceed, we introduce the so-called feasibility condition, which determines whether distance $\delta_{x}$ can be covered within a certain number of moves.

Feasibility condition. For a fixed number $t_{x}$ of moves, we can cover a maximum distance $\delta_{\max }\left(t_{x}\right)$ by accelerating as long as possible (namely for $\left(t_{x}+s_{x}^{\prime}-s_{x}\right) / 2$ steps) and then by decelerating enough to reach $s_{x}^{\prime}$ (namely for $\left(t_{x}-s_{x}^{\prime}+s_{x}\right) / 2$ steps). Analogously, the minimum distance $\delta_{\min }\left(t_{x}\right)$ that can be covered with a certain number $t_{x}$ of moves is obtained by decelerating as long as possible (namely for $\left(t_{x}-s_{x}^{\prime}+s_{x}\right) / 2$ steps) and then by accelerating enough to reach $s_{x}^{\prime}$ (namely for $\left(t_{x}+s_{x}^{\prime}-s_{x}\right) / 2$ steps); see Figure 2a. Note that in our calculations we assume that the parities of the distances and the differences of the speeds are appropriate, otherwise we have to slightly adjust them, which can easily be done.

If $\delta_{\min }\left(t_{x}\right) \leq \delta_{x} \leq \delta_{\max }\left(t_{x}\right)$, then it is feasible to cover distance $\delta_{x}$ in $t_{x}$ moves (we refer to this condition as feasibility condition). In particular, we know that the route realizing distance $\delta_{x}$ resides between the two routes realizing distances $\delta_{\min }\left(t_{x}\right)$ and $\delta_{\max }\left(t_{x}\right)$. So, in order to realize distance $\delta_{x}$ in $t_{x}$ moves, we have either to accelerate or decelerate for a certain amount of steps, followed by some steps where the speed remains unchanged and finally decelerate or accelerate to reach speed $s_{x}^{\prime}$. This gives rises to three different cases, which can be computed by solving the corresponding quadratic equations. Note that during the second period where the speed should remain unchanged, we might have to change it once by one unit, in order to reach precisely the desired distance $\delta_{x}$; see Figure 2c.

Finding the smallest number of moves under the feasibility condition. In the following, we seek for the smallest $t_{x}$ for which the feasibility condition holds. Note that $\delta_{\max }\left(s_{x}^{\prime}-s_{x}\right)=$ $\delta_{\min }\left(s_{x}^{\prime}-s_{x}\right)$. Let $t_{x}^{*}$ be the number of moves when the car brakes down to zero speed and then accelerates again to speed $s_{x}^{\prime}$ (observe that in this case the car moves only "forward"). We will consider two cases: (i) $\tau_{x} \leq t_{x}^{*}$ and (ii) $\tau_{x}>t_{x}^{*}$.

First consider Case (i). This case is possible, only if $\delta_{\max }\left(t_{x}^{*}\right) \geq \delta_{x}$ (which implies that $\alpha_{x}<\delta_{x}$ holds; if $\alpha_{x}>\delta_{x}$, then $\tau_{x}$ must be larger than $\left.t_{x}^{*}\right)$. Let $t_{x}^{+} \leq t_{x}^{*}$ be the smallest value for which $\delta_{\max }\left(t_{x}^{+}\right) \geq \delta_{x}$ holds (note that $t_{x}^{+}$can be computed by solving the corresponding quadratic equation). If additionally $\delta_{\min }\left(t_{x}^{+}\right) \leq \delta_{x}$ holds, then by the feasibility condition it follows that $\tau_{x}=t_{x}^{+}$. Otherwise, $\tau_{x}$ cannot be smaller than $t_{x}^{*}$ (that is, Case (ii) applies).

From the above, it follows that in Case (ii) either $\delta_{\max }\left(t_{x}^{*}\right)<\delta_{x}$ or $\delta_{\min }\left(t_{x}^{+}\right)>\delta_{x}$ holds. To compute the minimum distance $\delta_{\min }\left(t_{x}\right)$ that can be covered by a car in $t_{x}$ steps when $t_{x}>t_{x}^{*}$, we observe that the car has to reverse its direction. This subdivides the movement into four phases: The car has to brake from speed $s_{x}$ to zero speed, further brake to a "negative" speed, then accelerate to zero speed and then to $s_{x}^{\prime}$; see Figure $2 \mathrm{~b}$. Only in the 


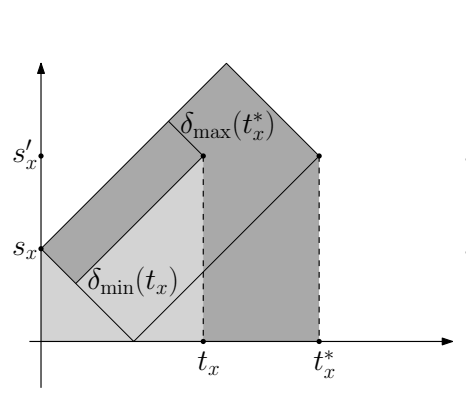

(a)

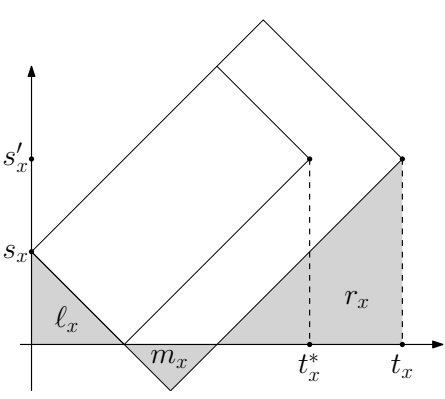

(b)

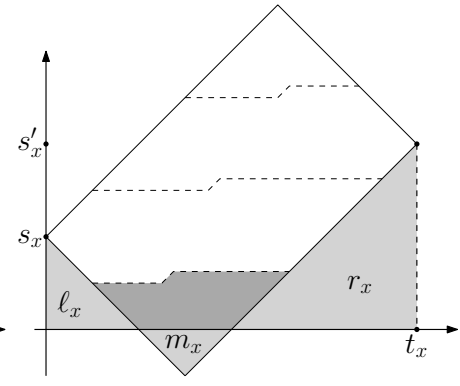

(c)

Figure 2 In the charts, the $x$-axis corresponds to the number of moves, the $y$-axis to the possible speeds. The light gray shaded areas indicate the corresponding distances for $\delta_{\min }\left(t_{x}\right)$. Figures (a) and (b) illustrate the configurations corresponding to $\delta_{\min }$ and $\delta_{\max }$ values for $t_{x}<t_{x}^{*}$ and $t_{x}>t_{x}^{*}$, respectively. Figure (c) illustrates the different cases that might arise when computing the route realizing $\delta_{x}$ when the feasibility condition holds for $t_{x}$. The dark gray region corresponds to the difference between $\delta_{x}$ and $\delta_{\min }\left(t_{x}\right)$ for one particular case.

first and the last phases the car moves from $p$ towards $p^{\prime}$. The distance covered in these two phases is fixed. In the first phase there are $s_{x}$ steps of braking, which correspond to a distance of $s_{x}\left(s_{x}+1\right) / 2$. In the last phase there are $s_{x}^{\prime}$ steps of accelerating from zero, which correspond to a distance of $s_{x}^{\prime}\left(s_{x}^{\prime}+1\right) / 2$. Let $\ell_{x}$ and $r_{x}$ be these two distances. To perform $t_{x}$ step in total, we have to also perform $t_{x}-s_{x}-s_{x}^{\prime}$ steps in negative, which corresponds to a distance of $\left(t_{x}-s_{x}-s_{x}^{\prime}\right)\left(\left(t_{x}-s_{x}-s_{x}^{\prime}\right) / 2+1\right)$ that we denote by $m_{x}$; see Figure $2 \mathrm{~b}$. It follows that: $\delta_{\min }\left(t_{x}\right)=\ell_{x}+r_{x}-m_{x}$.

Let $t_{x}^{-} \geq t_{x}^{*}$ be the smallest value for which $\delta_{\min }\left(t_{x}^{-}\right) \leq \delta_{x}$ holds (note that $t_{x}^{-}$can be computed by solving the corresponding quadratic equation). If $\delta_{x} \leq \delta_{\max }\left(t_{x}^{-}\right)$, then by the feasibility condition we have that $\tau_{x}=t_{x}^{-}$. If this is not the case (that is, $\left.\delta_{x}>\delta_{\max }\left(t_{x}^{-}\right)\right)$, then we have to increase the value of $t_{x}^{-}$until $\delta_{x} \leq \delta_{\max }\left(t_{x}^{-}\right)$. Note that for this particular value of $t_{x}^{-}$, it will also hold that $\delta_{\min }\left(t_{x}^{-}\right) \leq \delta_{x}$, as $\delta_{\min }$ is a decreasing function. In the following, we prove the correctness of our approach.

- Lemma 1. The minimum number of moves computed under the feasibility condition ensures that the car does not crash.

Proof. We prove the claim for the movement between two consecutive landing regions, which are connected, say w.l.o.g., by a vertical track segment as illustrated in Figures 1 and 2. Clearly, only the two vertical sides of the track are critical in this case. As discussed above, the movement might consist of one or three monotone parts, depending on Cases (i) or (ii). In Case (i), the movement consists of only one monotone part. Since there is no vertical side of the track between the two landing points of that part, the car will not crash. In Case (ii), the movement consists of three monotone parts. The first part consists of a braking phase ending with speed zero. Here, the choice of the landing point in the first landing region ensures that the car will not crash. Symmetrically the same holds for the third part, since it only consists of an acceleration phase from speed zero to the final speed. Since the endpoint of the first part and the starting point of the third part form the endpoints of the second part, and since the second part is also monotone, the car will not collide with vertical sides of the track. 
Coordinating the number of moves in both directions. Next, we discuss how to coordinate the moves in $x$ - and $y$-direction so to become same. Assume w.l.o.g. that $\tau_{y}<\tau_{x}$. In this case, we set $\tau_{y}$ to be equal to $\tau_{x}$ and check whether the feasibility condition holds, that is, whether $\delta_{\min }\left(\tau_{y}\right) \leq \delta_{y} \leq \delta_{\max }\left(\tau_{y}\right)$. In the positive case, we have managed to coordinate the moves in $x$ - and $y$-direction. In the negative case, $\delta_{y}<\delta_{\min }\left(\tau_{y}\right)$ must hold. So, we have to search for a new minimum value of $\tau_{y}$ to the right of $t_{y}^{*}$ (which corresponds to Case (ii) in the description given above), that is, $\tau_{y}$ has to be at least as large as $t_{y}^{-}$(and larger than $\tau_{x}$, as well). We do so and then we proceed by swapping the roles of $\tau_{x}$ and $\tau_{y}$ (to adjust $\tau_{x}$ to the new value of $\tau_{y}$ ). In worst case, $\tau_{x}$ and $\tau_{y}$ will become equal, only when both are larger than the minimum of $t_{x}^{-}$and $t_{y}^{-}$, that is, after at most two steps.

From above it follows that we can compute in constant time the minimum number of movements between two landing points of consecutive landing regions (for a certain pair of speed components).

\section{Computing the overall minimum number of moves}

Finally, we compute the minimum number of moves from a starting position to the finish line, when moving from one landing region to another. This is a shortest path problem where the weights on the edges of the underlying state graph have been computed before in the second phase of our algorithm. Since the graph is acyclic, we can do this in linear time using topological sort: In every landing region we have $O\left(W^{3 / 2}\right)$ points which are associated with $O(\sqrt{W}) \times O(\sqrt{W})$ of different values for the speed. Hence, in the state graph there exist $O\left(W^{5}\right)$ edges between any two adjacent landing regions. Since the number of landing regions of INDIANAPOLIS is constant, the state graph has $O\left(W^{5 / 2}\right)$ vertices and $O\left(W^{5}\right)$ edges. The above analysis together with Lemma 1, yields the following theorem.

- Theorem 2. The minimum number of moves for a single car in INDIANAPOLIS track can be computed in $O\left(W^{5}\right)$ time.

Note that the time complexity of our algorithm is independent of the size of the track (that is, independent of $\left.L_{1}, L_{2}\right)$. It only depends on $W$ which is usually a small constant. In particular, for $W=7$, which is a typical value for the size of the track, the number of vertices of the state graph does not exceed 700. We provide more details in Section 6.

\subsection{Extensions to more general tracks}

In the general scenario, we define a track to consist of horizontal and vertical track-segments, which overlap only at their common corner regions. As in the previous subsection, we will assume that our track is of uniform width $W$. Hence, each corner region is of size $W \times W$. We will further assume that consecutive corner regions are separated by at least $2 W$ units of distance in the horizontal or vertical direction, which also implies that no two corner regions overlap. In other words, we will assume that each track segment is of length at least $4 W$.

Our goal is to construct appropriate landing regions close to the corner regions, as we did in the previous subsection. If we can guarantee that the maximal speed in each direction is $O(\sqrt{W})$, then the landing regions will be of approximately the same size as the corresponding ones of the INDIANAPOLIS track. Note that in general this is not the case, i.e., if track segments of length $3 W$ are allowed. To see this, consider a staircase-shaped track, which consists of $s$ horizontal and vertical track segments of length exactly $3 W$, where $s$ is a sufficiently large integer; see Figure 3a. Assume w.l.o.g. that $W$ is an even integer and let the coordinates of the concave corners of this track be $(i W, i W), i=1,2, \ldots, s$. Then, a speed 


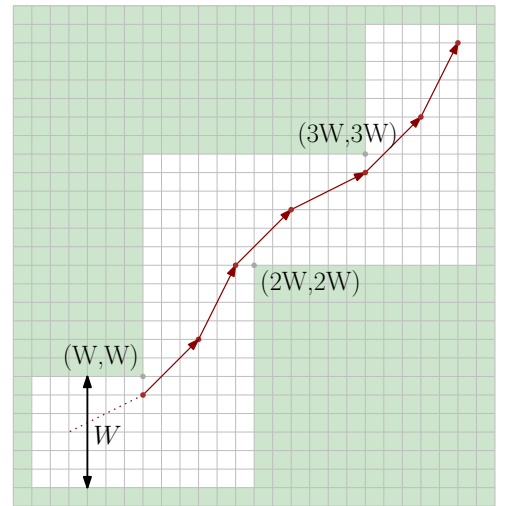

(a)

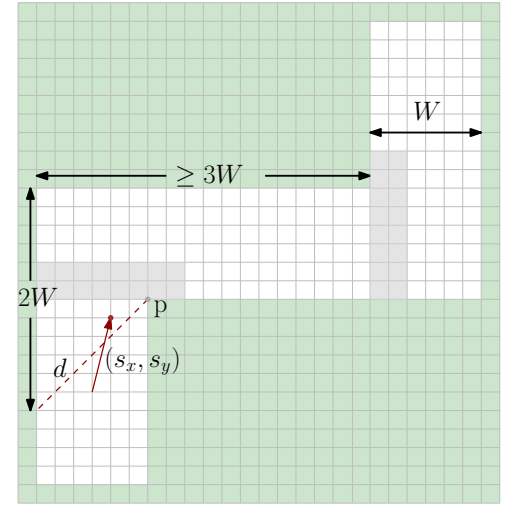

(b)

Figure 3 (a) Fast racing along a staircase-shaped track. (b) Entering a long track segment.

of $W / 2$ in either directions is possible: We start at point $(W, W-1)$ with speed $(W / 2, W / 2)$. Then, we continue with speeds $(W / 2+1, W / 2-1),(W / 2, W / 2),(W / 2-1, W / 2+1)$ and $(W / 2, W / 2)$. As we show in Figure 3a, the car stays nicely in the track, which implies that indeed a speed of $W / 2$ is possible in both directions. Note that such a high speed is not possible in the simple INDIANAPOLIS track.

Fortunately, if the segments of our track are of length at least $4 \mathrm{~W}$, then we can limit the speed to $O(\sqrt{W})$ in both directions. To see this, consider a corner region which is open at the bottom and the right hand side, where a vertical segment ends and a horizontal segment starts. Let $p$ be the concave corner at the corner region and let $d$ be the diagonal passing through $p$ with unit slope as shown in Figure $3 \mathrm{~b}$. When crossing this diagonal, the car will have a speed $\left(s_{x}, s_{y}\right)$, such that $s_{y}>s_{x}$ holds.

We now claim that $s_{x}, s_{y}=O(\sqrt{W})$. To prove our claim we first consider the vertical speed and the number, say $t$, of steps that we can make in the vertical direction. There exist two scenarios. Either $s_{y}$ is small enough to break before hitting the horizontal side of the track or $s_{y}$ is not small enough to avoid hitting the horizontal side of the track. In the latter case we can prevent hitting the side of the track after $t$ steps by reaching the next vertical segment of the track. Of course, in the first scenario it is reasonable to assume that the speed will be decreased as much as possible during these $t$ steps. Since the distance to the horizontal side of the track is at most $2 W$, it follows: $\left(s_{y}-1\right)+\ldots+\left(s_{y}-t\right) \leq 2 W$ if and only if $s_{y} t-t(t+1) / 2 \leq 2 W$.

It follows that if $s_{y} \leq \sqrt{4 W}$, then the car will be able to avoid hitting the horizontal side of the track. Since $s_{y}>s_{x}$, our claim follows. Next, we consider the second scenario. We can further assume w.l.o.g. that $s_{y}>\sqrt{4 W}$. Now, we ask how many steps can we perform in the vertical direction before hitting the horizontal side of the track? In this case, that the maximum number of steps that we can perform is no more than $2 W / s_{y}$. Hence, in this amount steps we have to be able to reach the next vertical segment of the track. W.l.o.g. we will suppose to accelerate in the horizontal direction for $t \leq 2 \mathrm{~W} / \mathrm{s}_{y}$ steps starting from speed $s_{x}$ and see how far we can go. The distance that we will cover in the horizontal direction is:

$$
\left(s_{x}+1\right)+\ldots+\left(s_{x}+t\right)=s_{x} t+t(t+1) / 2 \leq \frac{s_{x}}{s_{y}} \cdot 2 W+\frac{2 W^{2}}{s_{y}^{2}}+\frac{W}{s_{y}} \leq 2 W+\frac{W}{2}+\frac{\sqrt{W}}{2}<3 W .
$$

Since we have assumed that all track segments are of length at least $4 W$, we have obtained a contradiction. Therefore, $s_{y} \leq \sqrt{4 W}$ which also implies that $s_{x}=O(\sqrt{W})$. Now, that 
we know that the speeds are limited when crossing through the corner regions (provided that the track segments are long enough), we can determine the landing regions as described in Section 4.1. Since the distance from the diagonal defining $\left(s_{x}, s_{y}\right)$ to the actual landing region is at most $W$, it follows that the speed in both directions cannot change by more than $O(\sqrt{W})$. Hence, the size of the landing region remains approximately the same as before. We conclude that the complexity of our algorithm to move from one landing region to the next landing region is the same as the one of Theorem 2.

- Theorem 3. The minimum number of moves for a single car in a general track with rectilinear segments of length at least $4 W$ can be computed in $O\left(c W^{5}\right)$ time, where $c$ is the number of corners of the track.

\section{A competitive variant with limited view}

In this section, we adopt the concepts that we developed in Section 4.2 and we use them in a scenario which reflects some real-world property, namely the "limited view". This scenario arises naturally in several computer games with the driver's view perspective, namely, with view limited to a certain portion of the track. Here, we assume that we can only see from one corner region to the next corner along one single track segment, and we additionally see where the next segment continues (left or right). We have no information about the sequence or the lengths of the track segments that follow. In this regard, we develop five heuristic strategies (without any approximation guarantee) that lead to considerably more efficient implementations.

The rule we face is the following: We assume that we are currently landed on a certain landing point. From there, we can compute the necessary moves to all points in the next landing region. This can be accomplished using our techniques from the Section 4.2. Based only on this information, we choose one single position in the next landing region, where we go next. So, the selection of the new landing point is the subject of a strategy that we discuss in the following. When choosing the strategy, we have to distinguish between long track segments, where we are able to move from one side of the track to the other and short segments, where the entering positions to the new track will play a major role.

To cope with the limited view scenario, we develop a list of possible strategies. For the sake of simplicity, we will assume w.l.o.g. that the current track segment is horizontal followed by a vertical one and that the next landing region resides along a left turn. Our strategies for the remaining cases are defined symmetrically.

S.1 Drive safely: According to this strategy, we choose the middle-position of each landing region, which can be reached with the minimum number of moves from our current position (formally the middle possition of a landing region is identified with the middle row of it). We also choose a speed equal to at most $2 \sqrt{W}$ in both directions (safe speed), which ensures that the car will not collide, regardless of the sequence of track segments that follow. Since there exist $O(\sqrt{W})$ different middle positions in total, the time required to perform our choice is $O\left(W^{3 / 2}\right)$.

S.2 Drive carefully: Again, we choose the middle-position of each landing region, which can be reached with the minimum number of moves from our current position. However, we choose the speed to be equal to at most $\sqrt{W}$ in both directions (careful speed), which is half of the maximum speed of the previous strategy. Although the strategy is inspired by German in-town speed regulations, it has the effect that the car might avoid time-consuming $S$-shaped movements within one track segment as it is the case of Figure 2b. Note that the time required to perform our choice is still $O\left(W^{3 / 2}\right)$. 
S.3 Topmost with highest y-speed: According to this strategy, we aim for one point of the topmost row of the next landing region, so that the speed in the vertical direction is maximized (but still safe to avoid collisions). Since there exist $O(\sqrt{W})$ different points in the topmost row and for each of them the maximum $y$-speed is uniquely defined, it follows that only the values for the x-speed may vary and so we can find the position, which also requires the smallest number of moves from our current position, in $O(W)$ time.

S.4 Be fast between landing regions: According to this strategy, we aim for any point of the next landing region, so that the distance between the current and the next landing region is covered as fast as possible. If there exist more than one alternatives, then we choose the one with the highest y-speed. This choice can be done in $O\left(W^{5 / 2}\right)$ time.

S.5 Closest to the corner: According to this strategy, we aim for the point that is below and to the right of the internal corner of the turn, so that the $x$-speed at this point is one, while the $y$-speed is maximum. Since by construction the maximum speed at this point is uniquely defined, this choice can be done in $O(1)$ time.

Note that the running times that we gave above are restricted to pairs of landing regions. The total time complexity of our strategies is then subject to the total number of corners of the track. On the other hand, however, the theoretical performance of the strategies is a drastic improvement compared to the optimal algorithm (see the following proposition). We also note that the differences in the running times of the strategies might give a hint to the expected quality of the practical performance, which we discuss in Section 6 .

- Proposition 4. While the running time for computing the optimal solution is $O\left(\mathrm{cW}^{5}\right)$ from Theorem 3, the corresponding running time for $S .1$ and $S .2$ is $O\left(\mathrm{cW}^{3 / 2}\right)$, for $S .3$ is $O(\mathrm{cW})$, for $S .4$ is $O\left(c W^{5 / 2}\right)$, and for $S .5$ is $O(c)$, where $c$ is the number of corners of the track.

\section{Evaluation}

In this section, we present the results of the experimental evaluation of our algorithms. The experiment was performed on a Linux machine with four cores at 2,5 GHz and $8 \mathrm{GBs}$ of RAM. The implementations were in Java. Apart from our algorithms, we have also implemented algorithm $A^{*}$ [4], which is common in path-finding and graph traversal problems. This algorithm is similar to Dijkstra's algorithm. It starts from a single source, but in contrast to Dijkstra's algorithm, only the vertices that are mainly in the direction to the target are processed assuming that this direction is somehow known. The vertices of the graph, which are too far away from the assumed direction, are ignored. The direction is usually estimated by a heuristic function, e.g., by the Euclidean distance to the target. In such a way, algorithm $\mathrm{A}^{*}$ performs well in unknown search spaces.

\section{Optimal algorithms}

First, we experimentally compared our algorithm from Section 4 against algorithm A*. As a test set for our experiment, we used a simple (single-parameter) setting to get meaningful results. In particular, we used different instances of INDIANAPOLIS track, whose horizontal and vertical lengths were equal (that is, $L_{1}=L_{2}$ ) ranging from 64 to 224 units of length. The width $W$ of each track was set to 7 units of length.

Since the number of moves computed by both algorithms were equal (that is, both algorithms led to optimal solutions), we were mainly interested in comparing two aspects of these algorithms: (i) the total size of the underlying state graph (in terms of its number of edges) and (ii) the total time needed for computing the state graph together with finding the 


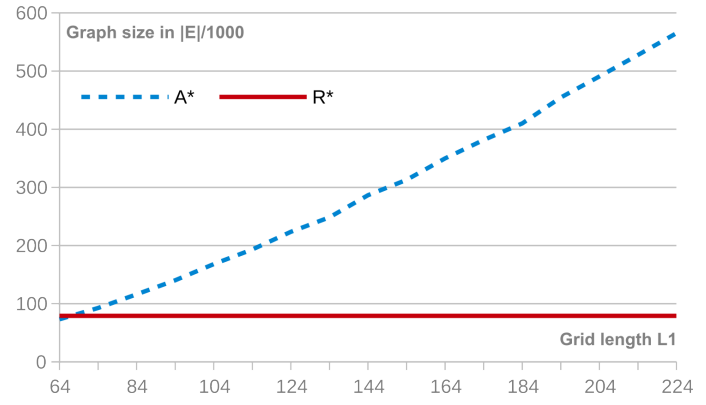

(a) Graph size against track size.

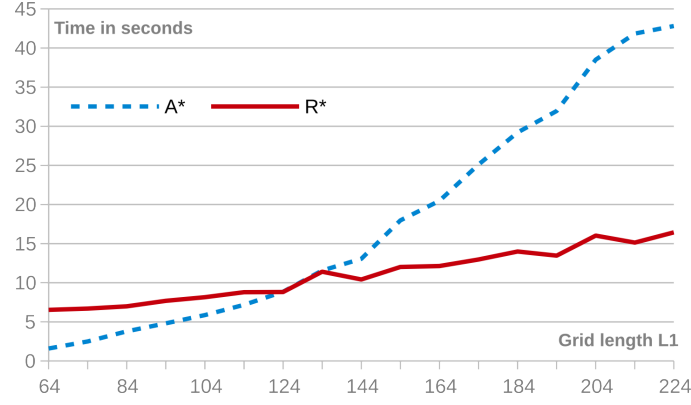

(b) Processing time against track size.

Figure 4 Statistics on different instances of the INDIANAPOLIS track. Comparing algorithm A* against our algorithm from Section 4 denoted by $R^{*}$ w.r.t. graph size and processing time.

optimal route. The results of our experimental comparison are illustrated in Figure 4. In the plots, the curve denoted by $\mathrm{R}^{*}$ stands for results of our algorithm from Section 4 . The curve denoted by $\mathrm{A}^{*}$ corresponds to the results obtained from algorithm $\mathrm{A}^{*}$.

It is eye-catching, that our algorithm uses a graph that is by far smaller than the corresponding graph used by algorithm A* (see Figure 4a). As expected, the size of the graph of algorithm $\mathrm{A}^{*}$ increases as the size of the track increases (ranging from 73.205 to 565.212 edges in our experiment). On the other hand, the size of the state graph in our algorithm was constant in our experiment, because the width of the track was fixed (recall that the size of the state graph of our algorithm is independent of the dimensions of the track; it only depends on the width of the track). More precisely, its number of edges was constantly 79.056 .

In Figure 4b, the computation time needed for computing the state graph together with finding the optimal route is plotted against the size of the tracks. More precisely, the computation time our algorithm is 6 seconds for a $64 \times 64$ track and increases slightly up to 16 seconds for larger tracks. On the hand, algorithm A* outperforms our algorithm for relatively small tracks of size at most $124 \times 124$. For larger tracks, however, it requires significantly more time (up to 43 seconds). Observe that the computation time of our algorithm increases as the size of the track increases, although the state graph remains of the same size. In our prototype, the calculations described in Section 4 are not optimally implemented and thus require more time for larger tracks.

\section{Limited view scenario}

In the second phase of our experimental evaluation, we compared strategies S.1,.., S.5 from Section 5 against the algorithm from Section 4, which leads to optimal solutions. As a test set for our experiment, we used different instances of STAIRCASE track, which consists of alternating horizontal and vertical track segments (see, e.g., Figure 3). For our experiment each instance of the STAIRCASE track had 10 stairs (that is, each track had 11 horizontal track segments and 10 vertical track segments). In addition, the horizontal and vertical track segments were of equal lengths ranging from $4 \mathrm{~W}$ to $20 \mathrm{~W}$ units of length, where the width $W$ of the tracks was set again to 7 units of length. The results of our experimental comparison are illustrated in Figure 5, where the number of moves required by each strategy is plotted against the track size. In the plots, the curve denoted by $\mathrm{R}^{*}$ stands for results of our algorithm from Section 4. The curves denoted by S.1,.., S.5 corresponds to the results obtained from the corresponding strategies from Section 5. 


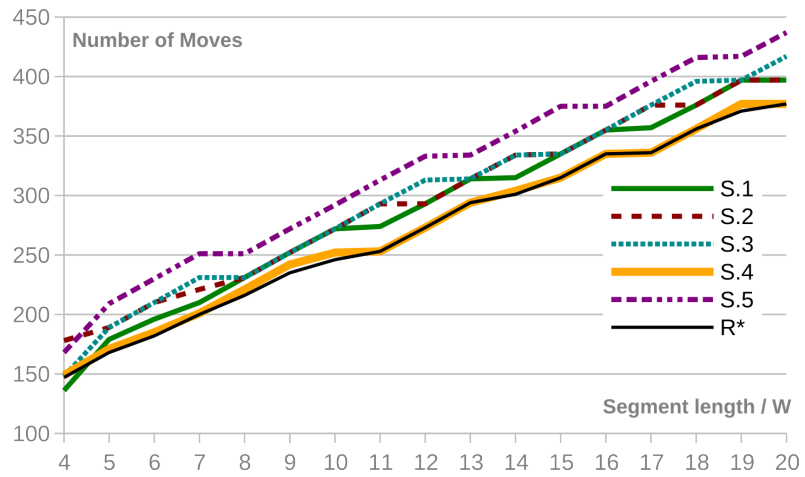

Figure 5 Statistics on different instances of the STAIRCASE track. Comparing our algorithm from Section 4 denoted by $\mathrm{R}^{*}$ against strategies $\mathrm{S} .1, \ldots$, S.5 w.r.t. the number of required moves.

It is clear that strategy S.4 (i.e., be fast between landing regions) outperforms the other ones as the length of the track segments increases. This is not surprising, since strategy S.4 seeks in covering the horizontal and the vertical track segments of the track as fast as possible. So, the longer these segments are, the more options this strategy has to cover the respective distances faster (and hence to win). Note also that strategy S.4 tends to have the same performance as the one of the optimal algorithm of Section 4 for tracks with long track segments. On the other hand, strategies S.1 and S.2 (i.e., drive safely and drive carefully, respectively) have, more or less, the same performance that is slightly better than the corresponding one of strategy S.3 (i.e., topmost with highest y-speed). Strategy S.5 (i.e., closest to the corner) is by far the one with worst performance, that is, it requires the maximum number of moves for the vast majority of the tracks of our experiment.

So, there seems to be a hierarchy between the strategies as the length of the track segments increases: S. $\rightarrow$ S. $1 \rightarrow$ S.2 $\rightarrow$ S. $3 \rightarrow$ S.5 from the best one to the worst one. This hierarchy is, up to a certain point, expected if one carefully observes the sizes of the graphs constructed by each strategy in order to compute the number of moves towards the next landing region (the higher a strategy is in the hierarchy, the larger is the graphs it uses), e.g., strategy S.4, which is the winning strategy, is the one that builds the largest graphs (and therefore takes into account more options). Strategies S.1 and S.2, that follow S.4 in the hierarchy, use graphs of comparable sizes. Hence, both have comparable performance, which is slightly better than the ones of S.3 and S.5 (but worse than the one of S.4).

An illustration of the routes of all strategies on a specific instance of the STAIRCASE track consisting of two stairs in total is given in Figure 6 . The width $W$ of this track is 7 (that is, equal to the one we used in our experimental evaluation; refer to Section 6). The length of the horizontal and vertical track segments of this track equal to $4 W$ units of length. In Figure 6, we have highlighted by large dots the positions of the cars after their 10th and 20th move. It is eye-catching, that strategy S.4 (i.e., be fast between landing-regions; yellow-colored in Figure 6) outperforms all other ones. Also, observe that strategies S.1 and S.2 (i.e., drive safely and drive carefully; green- and red-colored, respectively) have, more or less, the same performance as stated earlier.

\section{Conclusions}

We developed and experimentally evaluated efficient algorithms for single player scenarios of RACETRACK. We considered variants where in one case the whole track is supposed to be 


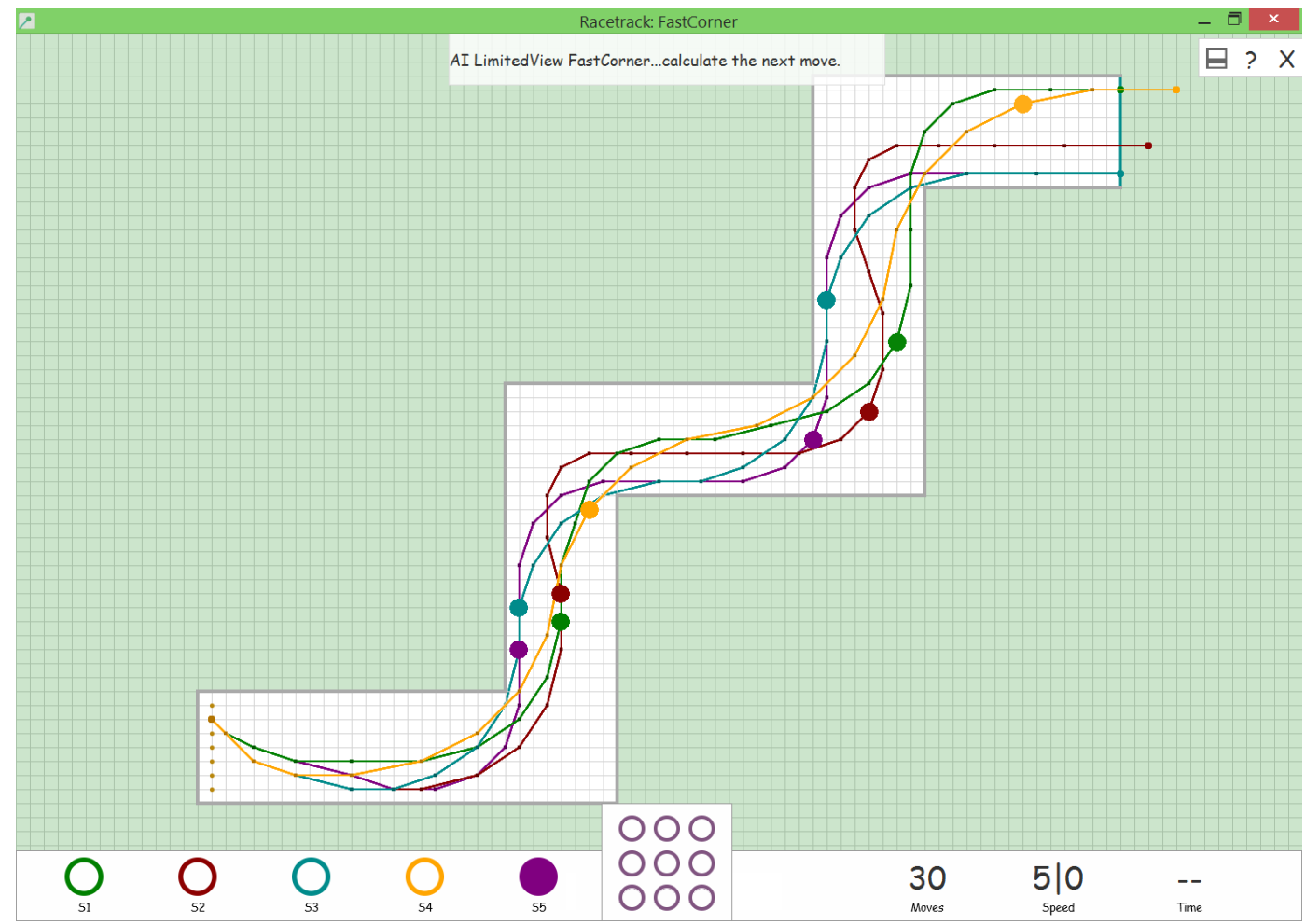

Figure 6 Illustration of the five strategies in the STAIRCASE track. The positions of the cars after their 10th and 20th move are highlighted by large dots.

known, or in another case the track is unknown except for these parts that are visible during the race (limited view). However, we restricted ourselves into discretized versions of the game and we used simple assumptions to make our algorithmic ideas applicable. In this regard, we conclude with the following open problems: (i) Apply the landing regions approach to scenarios with relatively short track segments (e.g., with overlapping corner regions) or to more challenging scenarios involving more than one player. (ii) Prove differences between overall optimal results and results from strategies under limited view. (iii) Prove quality bounds for different strategies. (iv) Develop efficient approaches for more general tracks.

Acknowledgments. This work has been supported by DFG grant Ka812/17-1. The authors would like to thank Stefan Feil, Denis Heid, Tobias Kaulich, and Sotirios Pavlidis for developing the user interface of our prototype.

\section{References}

1 Kristian Ahlmann-Ohlsen. Applying binary decision diagrams to solve the shortest path problem in vectorrace, 2005.

2 Jeff Erickson. How hard is optimal racing?, 2009. URL: http://3dpancakes.typepad. com/ernie/2009/06/how-hard-is-optimal-racing.html.

3 Martin Gardner. Mathematical games - Sim, chomp and race track: new games for the intellect (and not for lady luck). Scientific American, 228(1):108-115, 1973. 
4 Peter E. Hart, Nils J. Nilsson, and Bertram Raphael. A formal basis for the heuristic determination of minimum cost paths. IEEE Trans. Systems, Science and Cybernetics, SSC-4(2):100-107, 1968.

5 Markus Holzer and Pierre McKenzie. The computational complexity of racetrack. In Paolo Boldi and Luisa Gargano, editors, FUN 2010, volume 6099 of LNCS, pages 260-271. Springer, 2010.

6 Robert Olsson and Andreas Tarandi. A genetic algorithm in the game racetrack, 2011.

7 Jakob Schmid. VectorRace - finding the fastest path through a two-dimensional track, 2005. URL: http://schmid.dk/articles/vectorRace.pdf.

8 Wikipedia. Racetrack (game). URL: https://en.wikipedia.org/wiki/Racetrack_game. 